\title{
QUIMIOLUMINISCENCIA ELECTROGENERADA DEL LUMINOL USANDO ELECTRODOS DE BAJO COSTO
}

\author{
Guillermo Salgado* y José Navarrete
}

Facultad de Educación, Universidad Finis Terrae, Pedro de Valdivia, 1646, Santiago - Chile

Carlos Bustos, Cristián Sánchez y Ricardo Ugarte

Instituto de Química, Universidad Austral de Chile, Casilla 567, Valdivia - Chile

Recebido em 20/10/04; aceito em 6/6/05; publicado na web em 25/11/05

\begin{abstract}
ELECTROGENERATED CHEMILUMINESCENCE OF LUMINOL USING LOW-COST ELECTRODES. The purpose of the study was to observe eletrogenerated chemiluminescence (ECL) of luminol using different materials as electrodes such as wires, nails, coins, razor blades, etc. Based on the experimental observations and the mechanisms proposed in the literature, students should be capable of analyzing their results in order to understand the phenomena studied. Students could then elaborate a mechanism of action consistent with the experimental results which could rationalize the formation of intermediates in the reaction and the dependence of ECL on solution $\mathrm{pH}$ in addition to other points of interest.
\end{abstract}

Keywords: chemiluminiscence; chemical education; demonstrations experiments.

\section{INTRODUCCIÓN}

La electroquimioluminiscencia (también llamada quimioluminiscencia electrogenerada, ECL) es básicamente un medio para convertir energía eléctrica en energía radiativa. Es un proceso que involucra la generación de especies en la superficie del electrodo, que eventualmente participan en reacciones de transferencia electrónica conducentes a la formación de estados excitados que emiten luz. Por ejemplo, la aplicación de una diferencia de potencial a un electrodo en presencia de $\mathrm{Ru}(\mathrm{bpy})_{3}{ }^{2+}$ (bpy $=2,2$ '- bipyridine) da como resultado emisión de fotones y permite la detección del emisor a muy bajas concentraciones $\left(\leq 10^{-11} \mathrm{~mol} \mathrm{~L}^{-1}\right)^{1}$. El empleo de estas sustancias, como marcadores en biomoléculas, ha permitido implementar técnicas de inmunoensayo y de análisis de DNA ${ }^{2,3}$, además, es posible su uso en la investigación de las características de la superficie electródica ${ }^{4}$, técnica que se complementa con estudios de microscopía electrónica de barrido, de efecto túnel, etc.

\section{MECANISMOS GENERALES DE REACCIÓN}

\section{Aniquilación}

Los primeros estudios detallados sobre ECL involucraron reacciones de transferencia electrónica entre especies oxidadas y reducidas $\left(\mathrm{Ru}(\mathrm{bpy})_{3}{ }^{3+} \mathrm{y} \mathrm{Ru}(\mathrm{bpy})_{3}{ }^{+}\right)$, generadas en un electrodo de platino, por pulsos alternos de potencial $(0,2 \mathrm{~Hz})$, a partir de disoluciones de $\mathrm{Ru}(\mathrm{bpy})_{3}{ }^{2+}$ en acetonitrilo ${ }^{5}$.

Un mecanismo general de aniquilación es el siguiente:

$$
\begin{aligned}
& \mathrm{A} \rightarrow \mathrm{O}+\mathrm{e}^{-} \\
& \mathrm{B}+\mathrm{e}^{-} \rightarrow \mathrm{R} \\
& \text { (Oxidación en el electrodo) } \\
& \mathrm{A}^{*}\left(\text { ó B }^{*}\right) \rightarrow \mathrm{A}(\text { ó B) }+ \text { hv (Emisión de luz) }
\end{aligned}
$$

El potencial del electrodo de trabajo oscila rápidamente entre dos valores diferentes para generar la especies oxidada, O, y reducida, $\mathrm{R}$, (Ecs 1 y 2 , respectivamente, donde $\mathrm{O}$ y $\mathrm{R}$ podrían eventualmente representar especies radicalarias), que reaccionarán en las proximidades de la superficie del electrodo para formar el estado emisor (Ec 3). En relación al complejo de rutenio, $\mathrm{A}=\mathrm{B}$ y la especie excitada representado por $\mathrm{Ru}(\mathrm{bpy})_{3}{ }^{2+*}$ emite un fotón, $\mathrm{h} \frac{1}{2}$, al decaer a su estado basal. El estado excitado formado en esta reacción ECL es similar al que se obtiene en experimentos de fotoluminiscencia (PL). En PL un electrón es fotoexcitado produciéndose una transición por transferencia de carga del metal al ligando $\left(\pi \rightarrow \pi^{*}\right)$. Si la energía de excitación no es disipada o transferida, cruce intersistema puede ocurrir para formar el estado triplete más bajo del $\mathrm{Ru}(\mathrm{bpy})_{3}{ }^{2+*}$ el cual emitirá. La transición por transferencia de carga puede ser provocada en ECL si un electrón es transferido a un orbital $\pi^{*}$ de uno de los ligantes bipyridine. $\mathrm{Ru}(\mathrm{bpy})_{3}{ }^{2+*}$ puede, entonces, decaer al estado basal produciendo la misma luminiscencia que en PL.

Es preciso distinguir ECL de quimioluminiscencia (CL), si bien ambos involucran la producción de luz por especies que sufren reacciones de transferencia de carga altamente energéticas, sin embargo, en CL la luminiscencia es iniciada y controlada por la mezcla de reactivos y cuidadosa manipulación del flujo. En ECL, la luminiscencia es iniciada y controlada por la aplicación de un voltaje en un electrodo.

\section{Coreactante}

Un coreactante es una especie que luego de oxidarse o reducirse en la superficie del electrodo, produce por etapa química un intermediario que puede reaccionar con un luminóforo ECL para generar una especie excitada. Por ejemplo, el ión oxalato fue el primer coreactante descubierto ${ }^{6}$ y se postula que produce el fuerte reductor $\mathrm{CO}_{2}^{-}{ }^{-}$luego de su oxidación en disolución acuosa:

$\mathrm{C}_{2} \mathrm{O}_{4}^{2-} \rightarrow\left(\mathrm{C}_{2} \mathrm{O}_{4}^{--}\right) \rightarrow \mathrm{CO}_{2}^{--}+\mathrm{CO}_{2}+\mathrm{e}^{-}$

El potencial de oxidación impuesto sobre el electrodo de trabajo puede también oxidar a un luminóforo A (donde A es, por ejemplo, $\left.\mathrm{Ru}(\mathrm{bpy})_{3}^{2+}\right)^{\mathrm{ref}}$.

$\mathrm{A} \rightarrow \mathrm{O}+\mathrm{e}^{-}$ 
$\mathrm{O}$ y $\mathrm{CO}_{2}{ }^{-}$pueden entonces reaccionar para producir un estado excitado capaz de emitir luz.

$\mathrm{O}+\mathrm{CO}_{2}^{--} \rightarrow \mathrm{A}^{*}+\mathrm{CO}_{2}$

$\mathrm{A}^{*} \rightarrow \mathrm{A}+\mathrm{h} v$

\section{Luminiscencia catódica}

Emisión de luz también ha sido observada en electrodos de aluminio recubierto por películas delgadas de su óxido, inmersos en disoluciones de borato que contienen $\mathrm{Tl}$ (I) como luminóforo ${ }^{7}$. Se propone que este tipo de luminiscencia resulta de la reoxidación de los átomos de talio producidos catódicamente en la vecindad de la superficie del electrodo, requiriéndose para esto de la participación de coreactantes que en el cátodo se transforman en fuertes radicales oxidantes. Estos átomos de talio reoxidados (Tl (I) $\left.{ }^{*}\right)$ son los emisores de luz.

\section{LUMINOL}

\section{Quimioluminiscencia de luminol}

El luminol (2,3-Aminophthalhydrazide) es un compuesto orgánico que continua generando interés. CL de luminol $\left(\mathrm{LH}_{2}\right)$, en medio acuoso básico, ocurre en presencia de un reactivo oxidante $\left(\mathrm{H}_{2} \mathrm{O}_{2}, \mathrm{O}_{2}, \mathrm{HOCl}\right)$ y normalmente, de un metal de transición o ciertos iones inorgánicos, tal como ferricianuro, que actúan como catalizadores $^{8,9}$. En la reacción CL del luminol con peróxido de hidrógeno, el catalizador oxida al anión monobásico del luminol $\left(\mathrm{LH}^{-}, \mathrm{pK}=\right.$ 6,2 ) en dos etapas monoelectrónicas generando un intermediario azaquinona que es atacada por el anión del peróxido de hidrógeno $\left(\mathrm{HO}_{2}^{-}\right)$, produciendo un endoperóxido. Este último, pierde nitrógeno $\left(\mathrm{N}_{2}\right)$ y forma 3-aminoftalato en estado excitado, el cual decae a su estado fundamental emitiendo luz $(431 \mathrm{~nm})$.

También es posible obtener CL de luminol disuelto en dimetilsulfóxido ${ }^{8}$; en estas condiciones la reacción no necesita de peróxido de hidrógeno ni de catalizadores, sólo se precisa de oxígeno y de una base fuerte como el terc-butóxido de potasio. En estas condiciones el oxidante debe de ser el oxígeno. Al igual que en medio acuoso básico, la formación de azaquinona es considerada como etapa intermedia. Esta última, es atacada por el oxígeno o por el radical superóxido generándose un endoperóxido, cuya descomposición conduce finalmente a la emisión de luz (502nm) por formación del 3-aminoftalato en estado excitado.

\section{Electroquimioluminiscencia de luminol}

ECL en disoluciones acuosas alcalinas de luminol fue reportado por primera vez en 1929 por N. Harvey, al imponer $+2.8 \mathrm{~V}$ a través de dos electrodos inmersos en la disolución. Observó luminiscencia anódica con distintos materiales usados como ánodo y luminiscencia catódica solamente si el material usado como cátodo era mercurio ${ }^{10}$. Postuló que la luminiscencia anódica era inducida por el oxígeno generado electroquímicamente en el ánodo, y que el responsable de la emisión catódica era el peróxido de hidrógeno producido por la reducción electroquímica del oxígeno presente en la disolución ${ }^{11}$.

Varios mecanismos se han propuesto para la reacción ECL del luminol ${ }^{11-16}$.

En el mecanismo 1, el luminol deprotona en medio básico formando un anión que puede ser oxidado electroquímicamente en el ánodo para generar el intermediario azaquinona. Este último, es oxidado en presencia de peróxido de hidrógeno para producir el 3aminoftalato en estado excitado el cual decae emitiendo luz.

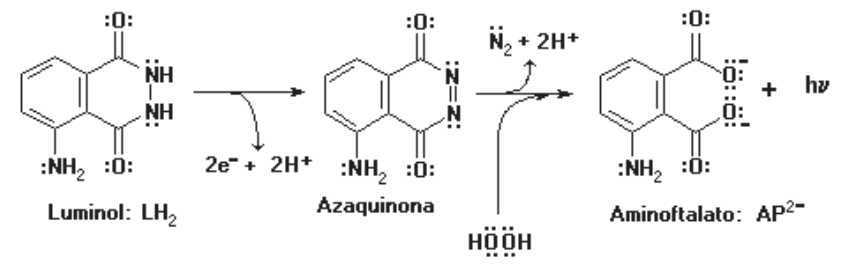

Mecanismo 1 propuesto para la ECL de luminol

Otro mecanismo coherente con los resultados experimentales es el que se detalla a continuación $n^{5}$ :

a - Reacciones de electrodo para luminol

$\mathrm{LH}^{-}+\mathrm{OH}^{-} \rightarrow \mathrm{L}^{--}+\mathrm{e}^{-}+\mathrm{H}_{2} \mathrm{O}$

$\mathrm{L}^{-}+4 \mathrm{OH}^{-} \rightarrow \mathrm{AP}^{2-}+\mathrm{N}_{2}+3 \mathrm{e}^{-}+2 \mathrm{H}_{2} \mathrm{O}$

b - Reacciones de electrodo para $\mathrm{H}_{2} \mathrm{O}_{2}$

$\mathrm{H}_{2} \mathrm{O}_{2}+\mathrm{OH}^{-} \rightarrow \mathrm{O}_{2}^{--}+\mathrm{e}^{-}+\mathrm{H}_{3} \mathrm{O}^{+}$

$\mathrm{O}_{2}^{\cdot-} \rightarrow \mathrm{O}_{2}+\mathrm{e}^{-}$

c - Reacciones en la disolución

$\mathrm{L}^{\cdot-}+\mathrm{O}_{2} \rightarrow \mathrm{O}_{2}^{\cdot-}+\mathrm{L}$

$\mathrm{L}^{\cdot-}+\mathrm{O}_{2}^{--} \rightarrow \mathrm{AP}^{2-}+\mathrm{N}_{2}+\mathrm{Luz}$

$\mathrm{H}_{2} \mathrm{O}+\mathrm{O}_{2}^{--} \rightarrow \mathrm{HO}_{2}^{-}+\mathrm{OH}^{-}$

Mecanismo 2 propuesto para la ECL de luminol

En el mecanismo 2, el radical $\mathrm{L}-$, $^{-}$producto de la oxidación monoelectrónica de $\mathrm{LH}^{-}$(8), reacciona con el radical superóxido, $\mathrm{O}_{2} \cdot$, que se genera a partir de la oxidación del peróxido de hidrógeno (10) o de la desactivación de L-- (12). El producto de esta reacción (13) es el emisor de fotones, 3-aminoftalato, $\mathrm{AP}^{2-}$.

En el presente trabajo se estudia cualitativamente el efecto de diferentes materiales usados como ánodo en la ECL de luminol. El objetivo principal es que en base a las observaciones experimentales y los mecanismos de reacción propuestos en literatura, los alumnos interpreten los resultados obtenidos, comprendan el concepto y el comportamiento de las reacciones de quimioluminiscencia electrogenerada.

\section{OBJETIVOS ESPECIFICOS}

Al término del experimento el alumno será capaz de: verificar que sin el paso de corriente eléctrica a través de la celda electroquímica no hay emisión de luz; verificar que la emisión de luz depende del material usado como ánodo y del tratamiento previo a que es sometido; comprender la función del peróxido de hidrógeno como coreactante y proponer un posible mecanismo de reacción que sea plausible con lo observado.

\section{REACTIVOS}

Disolución fresca de hidróxido de sodio $0,1 \mathrm{~mol} \mathrm{~L}^{-1}$; disolución de luminol, preparada por mezcla de $0,2 \mathrm{~g}$ de luminol en $125 \mathrm{~mL}$ de $\mathrm{NaOH} 0,1 \mathrm{~mol} \mathrm{~L}^{-1}$; disolución de $\mathrm{H}_{2} \mathrm{O}_{2}$ de 10 volúmenes y disolución de $\mathrm{HNO}_{3}$ concentrado. 


\section{MATERIALES Y EQUIPOS}

Fuente de corriente continua de $6,0 \mathrm{~V}$ o pilas (baterías) comerciales de 1,5 V dispuestas en serie; agitador magnético; soportes universales $(2 \mathrm{U})$; pinzas tres dedos $(2 \mathrm{U})$; conectores de doble tornillos o mufas ( $2 \mathrm{U})$; conectores eléctrico caimán-caimán (2 U); vasos de precipitado $100 \mathrm{~mL}$; vasos precipitado $250 \mathrm{~mL}$; vasos precipitados $400 \mathrm{~mL}$; barras de agitación magnética; electrodos de diversos materiales; pipetas graduadas; pipetas de Pasteur y lija fina o abrasivo para metales.

\section{PROCEDIMIENTO EXPERIMENTAL}

Armar el equipo tal como se muestra en la Figura 1. Luego, tomar alícuotas de $60 \mathrm{~mL}$ de la disolución básica de luminol en distintos vasos de precipitado de $100 \mathrm{~mL}$. Conectar los cables conectores caimán-caimán a la fuente de poder y a los electrodos inmersos en la disolución básica de luminol. En cada experimento use como cátodo y ánodo el mismo material. En caso de utilizar una batería puede ser necesario soldar cables a los terminales antes de realizar la conexión. Agitar la disolución, apagar la luz, conectar la fem y observar el fenómeno ocurrido.

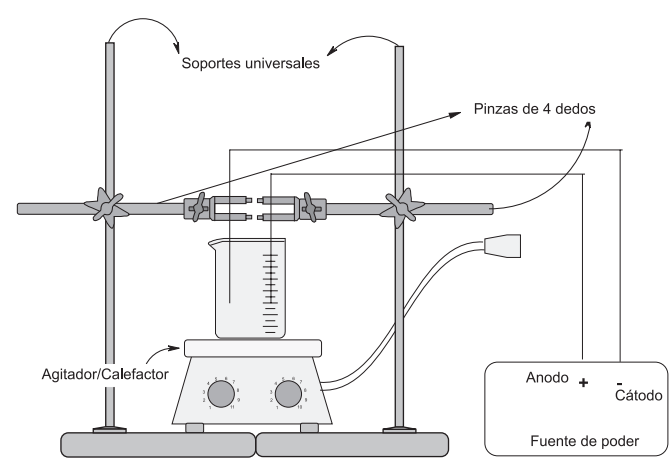

Figura 1. Montaje del equipo de electrólisis

\section{RESULTADOS EXPERIMENTALES}

Tabla 1. Resumen de los resultados experimentales de la ECL de luminol a 6,0 Volts

\begin{tabular}{|c|c|c|c|c|c|}
\hline Ensayo & Electrodos & $\mathrm{H}_{2} \mathrm{O}_{2}$ (gotas) & Tratamiento delelectrodo & Emisión & Observaciones \\
\hline $1 \mathbf{a}$ & Clavos acero & - & - & $(-)$ & $\begin{array}{l}\text { No se observa luminiscencia, la disolución adquiere } \\
\text { un color amarillo pálido. Hay burbujeo en la } \\
\text { superficie de ambos electrodos. }\end{array}$ \\
\hline $1 \mathbf{b}$ & Clavos acero & 10 & - & $(-)$ & 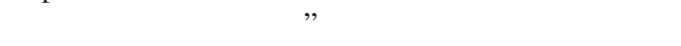 \\
\hline 1c & Clavos acero & - & $\begin{array}{l}\text { Pulido con lija fina } \\
\text { para metales }\end{array}$ & $(+)$ & $\begin{array}{l}\text { Se observa luminiscencia en toda la superficie del } \\
\text { ánodo, y abundante burbujeo en el cátodo. }\end{array}$ \\
\hline 1d & Clavos acero & 10 & $\begin{array}{l}\text { Pulido con lija fina } \\
\text { para metales }\end{array}$ & $(+)$ & Se observa ECL en el ánodo. \\
\hline $2 \mathbf{a}$ & Grafito $^{\mathbf{a}}$ & - & - & $(-)$ & $\begin{array}{l}\text { No se observa luminiscencia, la disolución adquiere } \\
\text { un color amarillo pálido. Hay burbujeo en la } \\
\text { superficie de ambos electrodos. }\end{array}$ \\
\hline $2 \mathbf{b}$ & Grafito & 10 & - & $(-)$ & $"$ \\
\hline 3a & Hojas afeitar ${ }^{\mathbf{b}}$ & - & - & $(+)$ & $\begin{array}{l}\text { Por ambas caras de la porción sumergida del ánodo } \\
\text { se observa ECL. La disolución adquiere un tono } \\
\text { amarillo oscuro. El ánodo queda recubierto de una } \\
\text { película de color café claro. }\end{array}$ \\
\hline $3 \mathbf{b}$ & Hojas afeitar & 10 & - & $(+)$ & Se observa ECL en el ánodo. \\
\hline $4 \mathbf{a}$ & Granallas Zn & - & - & $(-)$ & $\begin{array}{l}\text { No se observa luminiscencia, hay burbujeo en } \\
\text { ambas granallas. }\end{array}$ \\
\hline $4 b$ & Granallas Zn & 10 & - & $(-)$ & $"$ \\
\hline $5 \mathbf{a}$ & Moneda $\$ 10^{\mathrm{c}}$ & - & - & $(+)$ & $\begin{array}{l}\text { Se observa luminiscencia en el ánodo, en toda la } \\
\text { superficie de la moneda que está en contacto con la } \\
\text { disolución básica de luminol. Hay un abundante } \\
\text { burbujeo en el electrodo negativo. }\end{array}$ \\
\hline $5 \mathbf{b}$ & Moneda $\$ 10$ & 10 & - & $(+)$ & Se observa ECL en el ánodo. \\
\hline $6 \mathbf{a}$ & Amalgama $^{\text {d }}$ & - & - & $(+)$ & $\begin{array}{l}\text { Se observa luminiscencia en el ánodo, en toda la } \\
\text { superficie de la amalgama que está en contacto con } \\
\text { la disolución básica de luminol Hay un abundante } \\
\text { burbujeo en el electrodo negativo. }\end{array}$ \\
\hline $6 \mathbf{b}$ & Amalgama & 10 & - & $(+)$ & Se observa ECL en el ánodo. \\
\hline $7 \mathbf{a}$ & Cobre $^{e}$ & - & - & $(-)$ & $\begin{array}{l}\text { No se observa luminiscencia, hay burbujeo en } \\
\text { ambos electrodos. }\end{array}$ \\
\hline $7 \mathbf{b}$ & Cobre & 10 & - & $(-)$ & $"$ \\
\hline $7 c$ & Cobre & & $\mathrm{HNO}_{3}$ & $(-)$ & " \\
\hline $7 d$ & Cobre & 10 & $\mathrm{HNO}_{3}$ & $(-)$ & ” \\
\hline
\end{tabular}

a: los electrodos de grafito se obtuvieron de lápices de mina 2HB.; b: hojas de afeitar Gillete Platinum Plus; c: moneda de 10 centavos, acuñada en Brasil con fecha 1994; d: amalgamas utilizadas en obturaciones dentales, todas presentan formas irregulares y e: alambre de cobre para conexiones eléctricas. 


\section{RESULTADOS Y DISCUSIÓN}

Los antecedentes experimentales muestran que en el proceso de electrólisis de la disolución básica de luminol, no todos los electrodos ensayados producen la ECL. Además, es interesante notar que la emisión de luz puede ocurrir también en ausencia de peróxido de hidrógeno, $\mathrm{H}_{2} \mathrm{O}_{2}$, lo cual es evidencia de que en aquellos experimentos en que dicho reactivo estaba ausente, hubo generación in situ de $\mathrm{H}_{2} \mathrm{O}_{2}$ o en su defecto pudo formarse el anión superóxido, $\mathrm{O}_{2}{ }^{--}$directamente sobre la superficie del electrodo.

La discusión de las observaciones experimentales aquí encontradas, se efectuará tomando en consideración el mecanismo 2 de ECL, mencionado en la introducción. En disolución básica, $\mathrm{LH}_{2}$ sufre una ionización para generar LH', Fig. 2a. Luego, conforme a la reacción 8 , mediada por la fuente de poder, fem, se produce el radical $\mathrm{L}^{-}$, Fig 2b, que puede difundir desde la interfase electrododisolución y reaccionar con el anión superóxido, $\mathrm{O}_{2}^{--}$, producido por las reacciones 10, 12, y emitir luz conforme a la reacción 13. No obstante, si L-- no alcanza a difundir hacia el seno de la disolución, el radical se desactiva o apaga según la reacción 9. La cinética de la difusión del radical L-; evidentemente, estaría limitada por las características propias del material usado como electrodo, entre las que se puede mencionar la rugosidad superficial, la presencia de poros, las deformaciones, la presencia de películas pasivantes, etc. Esto opera en el sentido de que el fenómeno de adsorción de L“- sobre la superficie del electrodo, es un factor que limita la difusión, razón por la que no todos los electrodos ensayados conducen a la observación de luz.<smiles>Nc1cccc2c(=O)[nH]nc(Br)c12</smiles>

a: Anión $\mathrm{LH}^{-}$<smiles>Nc1cccc2c(Br)nnc(Br)c12</smiles>

b: Radical L'-
Figura 2. Estructuras propuestas para $\mathrm{LH}^{-}$y $\mathrm{L}^{\cdot}$

Por otro lado, la presencia de $\mathrm{H}_{2} \mathrm{O}_{2}$ en el medio de reacción genera el radical superóxido, $\mathrm{O}_{2}{ }^{-}$,según la reacción (3) que en caso de no encontrarse con $\mathrm{L}-$, puede también apagarse según la reacción 11. No obstante, el fenómeno de la ECL es posible también observarlo en ausencia de $\mathrm{H}_{2} \mathrm{O}_{2}$. En efecto, en disoluciones básicas de luminol no desaireadas, el oxígeno molecular, $\mathrm{O}_{2}$, puede generar el radical superóxido, $\mathrm{O}_{2}{ }^{-}$, conforme a la reacción 12 , necesario para que ocurra la reacción 13. También es posible que en el ánodo, dependiendo del potencial aplicado, ocurra la siguiente reacción:

$2 \mathrm{OH}^{-} \rightarrow \mathrm{H}_{2} \mathrm{O}_{2}+2 \mathrm{e}^{-}$

De este modo, la ECL operaría de un modo similar a como acontece cuando en el medio se incorpora disolución de $\mathrm{H}_{2} \mathrm{O}_{2}$.

Apoyándose en el análisis anterior, el alumno debería ser capaz de desarrollar el mecanismo 1 en una serie de etapas que contemplen la formación del anión monobásico del luminol, $\mathrm{LH}^{-}$, la generación electroquímica de azaquinona y su eventual reacción con $\mathrm{H}_{2} \mathrm{O}_{2}$ o con alguna de sus especies electrogeneradas, y finalmente la producción del 3-aminoftalato con la correspondiente emisión de fotones. Además, el mecanismo propuesto por el alumno, debe ser consecuente con todas las observaciones experimentales, como por ejemplo, evolución de $\mathrm{N}_{2}$ y la dependencia de la ECL con el pH de la disolución.

\section{REFERENCIAS}

1. Richter, M. M.; Chem. Rev. 2004, 104, 3003.

2. Wilson, R.; Kremeskötter, J.; Schiffrinn, D. J.; Wilkinson, J. S.; Biosens. Bioelectron. 1996, 11, 805.

3. Zhao, S.; Consoli, U.; Arceci, R.; Pfeiffer, J.; Dalton, W. S.; Andreff, M.; BioTechniques 1996, 21, 726.

4. Kukova, A. V.; Bykh, A. I.; Svir, I. B.; Fresenius J. Anal. Chem. 2000, 368, 439.

5. Tokel, N. E.; Bard, A. J.; J. Am. Chem. Soc. 1972, 94, 2862.

6. Rubinstein, I.; Bard, A. J.; J. Am. Chem. Soc. 1981, 103, 512.

7. Kulmala, S.; Ala-Kleme, T.; Vare, L.; Helin, M.; Lahtimer, T.; Anal. Chim. Acta. 1999, 398, 41

8. Albertin, R.; Arribas, M.; Bastos, E.; Röpke, S.; Sakai, P.; Sanches, A.; Stevani, C.; Umezu, I.; Yu, J.; Bader, W.; Quim. Nova 1998, 21, 772.

9. Bustos, C.; Salgado, G.; López, C.; Chem. Educator 2001, 6, 1.

10. Harvey, K. E.; J. Phys. Chem. 1929, 33, 1456.

11. Haapakka, K. E.; Kankare, J. J.; Anal. Chim. Acta 1982, 138, 263.

12. Kremeskötter, J.; Wilson, R.; Schiffrinn, D.; Luff, B. J.; Wilkinson, J. S.; Meas. Sci. Technol. 1995, 6, 1324.

13. Simison, S.; Pellicano, A.; Brust, M.; Schiffrin, D.; J. Electroanal. Chem. 1999, 470, 89.

14. Merenyi, G.; Lind, J. S.; J. Am. Chem. Soc. 1980, 102, 5830.

15 Witt, J.; Johnson, D.; Engstrom, R.; J. Electrochem. Soc. 1991, 138, 1637.

16. Wilson, R.; Schiffrin, D. J.; Anal. Chem. 1996, 68, 1254. 